\title{
CONTROL PROBLEMS GOVERNED BY A PSEUDO-PARABOLIC PARTIAL DIFFERENTIAL EQUATION
}

\author{
BY
}

LUTHER W. WHITE

\begin{abstract}
Let $G$ be a bounded domain in $R^{n}$ and $Q=G \times(0, T)$. We consider the solution $y(u)$ of the pseudo-parabolic initial-value problem

$$
\begin{gathered}
(1+M(x)) y_{t}(u)+L(x) y(u)=u \text { in } L^{2}(Q), \\
y(\cdot, 0 ; u)=0 \text { in } L^{2}(G),
\end{gathered}
$$

to be the state corresponding to the control $u$. Here $M(x)$ and $L(x)$ are symmetric uniformly strongly elliptic second-order partial differential operators. The control problem is to find a control $u_{0}$ in a fixed ball in $L^{2}(Q)$ such that (i) the endpoint of the corresponding state $y\left(\cdot, T ; u_{0}\right)$ lies in a given neighborhood of a target $Z$ in $L^{2}(G)$ and (ii) $u_{0}$ minimizes a certain energy functional. In this paper we establish results concerning the controllability of the states and the compatibility of the constraints, existence and uniqueness of the optimal control, existence and properties of Lagrange multipliers associated with the constraints, and regularity properties of the optimal control.
\end{abstract}

1. Introduction to the problem. In this paper we establish regularity properties of a control $u_{0}$ that is the solution of a problem that seeks to minimize a certain functional over a set of controls that are contained in a fixed ball in a Hilbert space and that steer their state functions to within a prescribed tolerance of a given target function. Here the state functions are solutions of a pseudo-parabolic initial-value problem. In the course of this study, we establish results concerning the controllability of pseudo-parabolic problems, compatibility of constraints, and properties of Lagrange multipliers associated with the constraints. While control systems governed by elliptic, parabolic, and hyperbolic equations have been studied extensively [2], [6], [9], those equations of Sobolev-Galpern type have as yet received little attention.

Let $G$ be a bounded domain in $\mathbf{R}^{n}$ and let $Q=G \times(0, T)$ where $T$ is finite. We assume that the boundary of $G$ is of class $C^{p}$ for $p>2$. We shall be concerned with several function spaces. Let $L^{2}(Q)$ and $L^{2}(G)$ denote the spaces of equivalence classes of square integrable functions on $Q$ and $G$. The norms in these spaces are denoted by $\|\cdot\|_{0,0}$ and $\|\cdot\|_{0}$ and inner products by

Received by the editors August 10, 1977 and, in revised form, March 10, 1978.

AMS (MOS) subject classifications (1970). Primary 49A20, 49B25.

Key words and phrases. Pseudo-parabolic equation, optimal control, Lagrange multiplier, controllability, Lagrangian, regularity, target set. 
$(\cdot, \cdot)_{0,0}$ and $(\cdot, \cdot)_{0}$, respectively. As is standard, we use $H^{k}(G)$ to denote the $k$ th order Sobolev space on $G$ with norm $\|\cdot\|_{k}$ and $H_{0}^{k}(G)$ to denote the completion with respect to $\|\cdot\|_{k}$ of the space of infinitely differentiable functions with compact support in $G$. For $k \geqslant 1$ the space $H_{0}^{1}(G) \cap H^{k}(G)$ is a closed subspace of $H^{k}(G)$. The spaces $L^{2}\left(0, T ; H_{0}^{1}(G) \cap H^{k}(G)\right)$ and $H^{1}\left(0, T ; H_{0}^{1}(G) \cap H^{k}(G)\right)$ are Hilbert spaces [4], [6] with norms $\|f\|_{k, 0}=$ $\left(\int_{0}^{T}\|f(\cdot, t)\|_{k}^{2} d t\right)^{1 / 2}$ and $\|f\|_{k, 1}=\left[\int_{0}^{T}\left(\|f(\cdot, t)\|_{k}^{2}+\|d f(\cdot, t) / d t\|_{0}^{2} d t\right)\right]^{1 / 2}$, respectively.

We consider $y$ in $L^{2}\left(0, T ; H_{0}^{1}(G) \cap H^{2}(G)\right)$, the solution of the pseudoparabolic Cauchy problem

$$
\begin{gathered}
(1+M(x)) y_{t}+L(x) y=u \quad \text { in } L^{2}(Q), \\
y(\cdot, 0)=0 \quad \text { in } L^{2}(G) .
\end{gathered}
$$

Here $M(x)$ and $L(x)$ are second-order partial differential operators on $G$ defined by

$$
M(x) \equiv-\sum_{i j=1}^{n} \frac{\partial}{\partial x_{i}}\left(m_{i j}(x) \frac{\partial}{\partial x_{j}}\right)+m(x)
$$

and

$$
L(x) \equiv-\sum_{i j=1}^{n} \frac{\partial}{\partial x_{i}}\left(l_{i j}(x) \frac{\partial}{\partial x_{j}}\right)+l(x) .
$$

We assume that $M$ and $L$ satisfy the following properties.

(i) The coefficients $m_{i j}$ and $l_{i j}$ belong to $C^{p-1}(\bar{G})$, and $m$ and $l$ belong to $C^{p-2}(\bar{G})$. Note this implies the existence of positive numbers $k_{m}$ and $k_{l}$ such that $((1+M) \varphi, \psi)_{0} \leqslant k_{m}\|\varphi\|_{1}\|\psi\|_{1}$ and $(L \varphi, \psi)_{0}<k_{l}\|\varphi\|_{1}\|\psi\|_{1}$.

(ii) The functions $m$ and $l$ satisfy $m(x) \geqslant 0$ and $l(x)>0$ for all $x$ in $G$.

(iii) The operators $M$ and $L$ are symmetric uniformly strongly elliptic on $G$. That is $m_{i j}(x)=m_{j i}(x)$ and $l_{i j}(x)=l_{j i}(x)$, and there exist constants $c_{m}>0$ and $c_{l}>0$ such that

$$
\sum_{i, j=1}^{n} m_{i j}(x) \xi_{i} \xi_{j} \geqslant c_{m} \sum_{i=1}^{n} \xi_{i}^{2} \text { and } \sum_{i j=1}^{n} l_{i j}(x) \xi_{i} \xi_{j} \geqslant c_{i} \sum_{i=1}^{n} \xi_{i}^{2}
$$

for each $x$ in $G$.

The control of (1.1) is to be exerted through the functions $u$ which are allowed to vary within a subset $U_{\text {ad }}$ in $L^{2}(Q)$. To denote the dependence of $y$ upon $u$, we write $y(u), y(\cdot, \cdot ; u)$, or $y(\cdot, t ; u)$. The set $U_{\text {ad }}$ contains those functions $u$ in a ball of radius $b$ in $L^{2}(Q)$ that steer the solutions of (1.1) in such a way that the traces $y(\cdot, T ; u)$ are within a prescribed tolerance $\rho$ of a given target function $Z$ in $L^{2}(G)$. When we define the sets

$$
N(0, b)=\left\{u \in L^{2}(Q):\|u\|_{0,0}<b\right\}
$$


and

$$
V(Z, \rho)=\left\{u \in L^{2}(Q):\|y(\cdot, T ; u)-Z(\cdot)\|_{0} \leqslant \rho\right\},
$$

we can write

$$
U_{\text {ad }}=N(0, b) \cap V(Z, \rho) .
$$

The control problem is to find a control $u_{0}$ in $U_{\text {ad }}$ that minimizes the energy functional (cf. (3.11)) given by

$$
K(u)=(u, y(u))_{0,0}
$$

over $U_{\mathrm{ad}}$. That is, find the element that solves the problem

$$
\text { minimize } K(u) \text { subject to }\|u\|_{0,0} \leqslant b \text { and }\|y(\cdot, T ; u)-Z(\cdot)\|_{0} \leqslant \rho \text {. }
$$

We interpret this problem as that of finding a norm bounded control that steers to a given target using the least energy.

In order for (1.6) to be meaningful, we must know that $U_{\mathrm{ad}}$ is nonempty. This leads to the consideration of the controllability of (1.1) and the compatibility of the control constraints. Establishing these results, we indicate the existence and uniqueness of the optimal control. Then we study properties of Lagrange multipliers associated with the constraints to establish the regularity properties for the optimal control. The relation of these results to those for parabolic problems will be the subject of subsequent papers.

2. Results on pseudo-parabolic equations. The pseudo-parabolic equation is a partial differential equation of Sobolev-Galpern type. It arises in the investigation of such physical problems as the flow of second-order fluids, the transfer of heat, and the emission of radiation from a gas. It has been studied extensively and results concerning the existence, uniqueness, growth, and regularity of its solution have been established. An extensive survey of the literature treating problems of Sobolev-Galpern type may be found in Carroll and Showalter [3].

We briefly discuss the properties of the solution of (1.1). Since $L$ and $M$ are isomorphisms of $H_{0}^{1}(G) \cap H^{2}(G)$ onto $L^{2}(G)$ [1], [4], [10], [11], the map $(1+M)^{-1} L$ is a bounded map of $H_{0}^{1}(G) \cap H^{2}(G)$ onto itself. Hence for each $\tau$ in $R$, we may define the map $E(\tau) \equiv \exp \left(-\tau(1+M)^{-1} L\right)$, and it too is an isomorphism of $H_{0}^{1}(G) \cap H^{2}(G)$ onto itself. The solution of (1.1) may be represented by

$$
y(\cdot, t ; u)=\int_{0}^{t} E(t-s)(1+M)^{-1} u(\cdot, s) d s
$$

where the integral is in the sense of Bochner [5], [13]. Several important properties are a consequence of (2.1) which we list for reference.

Proposition 2.1. For $u$ in $L^{2}(Q)$, define the map $t \rightarrow y(\cdot, t ; u)$ by $(2.1)$. Then, the following results are true. 
(1) The function $y(u)$ belongs to $L^{2}\left(0, T ; H_{0}^{1}(G) \cap H^{2}(G)\right)$.

(2) The map $t \rightarrow y(\cdot, t ; u)$ is strongly differentiable from $(0, T)$ into $H_{0}^{1}(G) \cap$ $H^{2}(G)$.

Furthermore, we have

$$
\begin{aligned}
y_{t}(\cdot, t ; u)= & -(1+M)^{-1} L \int_{0}^{t} E(t-s)(1+M)^{-1} u(\cdot, s) d s \\
& +(1+M)^{-1} u(\cdot, t)
\end{aligned}
$$

in $H_{0}^{1}(G) \cap H^{2}(G)$ for almost all $t$ in $(0, T)$.

REMARK 2.2. From this proposition, we see that the solution $y(u)$ is such that $y(u)$ and $y_{t}(u)$ are both in $L^{2}\left(\dot{0}, T ; H_{0}^{1}(G) \cap H^{2}(G)\right)$. Accordingly, we note that $y(u)$ is in the space $H^{1}\left(0, T ; H_{0}^{1}(G) \cap H^{2}(G)\right)$. As a consequence of Proposition 2.1 , we have the continuity result

COROllary 2.3. The mapping $u \rightarrow y(u)$ of $L^{2}(Q)$ into $H^{1}\left(0, T ; H_{0}^{1}(G) \cap\right.$ $\left.H^{2}(G)\right)$ is continuous. That is, there exists a positive constant $C$ dependent only on $T, M$ and $L$ such that $\|y(u)\|_{2,1}<C\|u\|_{0,0}$.

Another result that will be useful in our study concerns the growth of the solution of the initial-value problem

$$
\begin{gathered}
(1+M) y_{t}+L y=0 \quad \text { in } L^{2}(Q), \\
y(\cdot, 0)=\varphi(\cdot) \quad \text { in } H_{0}^{1}(G) \cap H^{2}(G) .
\end{gathered}
$$

The solution of $(2.3)$ is given by

$$
y(\cdot, t)=E(t) \varphi(\cdot)
$$

We state a result establishing the growth of $y$ presented in [10].

Proposition 2.4. The solution of (2.3) satisfies the following inequality

$$
\|y(\cdot, t)\|_{0}<\sqrt{k_{m}} e^{-t c_{1} / k_{m}}\|\varphi(\cdot)\|_{1} .
$$

In addition to (1.1), we shall also be concerned with pseudo-parabolic problems of the form

and

$$
\begin{gathered}
-(1+M) p_{t}+L p=u \quad \text { in } L^{2}(Q), \\
p(\cdot, T)=0 \text { in } L^{2}(G),
\end{gathered}
$$

$$
\begin{gathered}
-(1+M) q_{t}+L q=0 \text { in } L^{2}(Q), \\
q(\cdot, T)=(1+M)^{-1} \varphi(\cdot) \quad \text { in } H_{0}^{1}(G) \cap H^{2}(G) .
\end{gathered}
$$

The solution $p$ of (2.6) has a representation similar to that of $y$. The solution $q$ of (2.7), however, is given by

$$
q(\cdot, t)=E(T-t)(1+M)^{-1} \varphi(\cdot)
$$


for $0<t<T$. Continuity results analogous to those above also hold for these problems.

3. Formulation of the problem. As we indicated in $\$ 1$, the set $U_{\text {ad }}$ must be nonempty in order for the problem (1.6) to be meaningful. In this section we give conditions for this to be true. We begin by considering the controllability of the initial-value problem (1.1). That is, we show that the set defined by

$$
Y(T)=\left\{y(\cdot, T ; u): u \in L^{2}(Q)\right\}
$$

is a dense linear subspace of $L^{2}(G)$. At this point we depart from the standard proof that the subspace $Y(T)$ is dense in $L^{2}(G)$ given in [6] in favor of one that produces controls that steer close to points in $H_{0}^{1}(G) \cap H^{2}(G)$.

LEMMA 3.1. Let $\tilde{z}$ belong to $H_{0}^{1}(G) \cap H^{2}(G)$ and $\rho$ be a positive real number. Then if

$$
N \geqslant\left(k_{m} /-T c_{l}\right) \log \left(\left(\rho /\|\tilde{z}\|_{1}\right) \sqrt{1 / k_{m}}\right)
$$

the control

$$
\tilde{u}=L \tilde{z}+(N-1) L E(N t) \tilde{z}
$$

satisfies $\|y(\cdot, T ; \tilde{u})-\tilde{z}(\cdot)\|_{0}<\rho$.

Proof. Consider the initial-value problem (1.1) with $u$ replaced by

$$
\tilde{u}(\cdot, t)=L \tilde{z}(\cdot)+(N-1) L E(N t) \tilde{z}(\cdot)
$$

for some positive integer $N$. Using the representation (2.1) and performing the integration, we see that

$$
y(\cdot, t ; \tilde{u})=\tilde{z}(\cdot)-E(N t) \tilde{z}(\cdot) .
$$

We now use (3.4) and the estimate given in Proposition 2.4 to obtain the result. To this end, consider the problem

$$
\begin{gathered}
(1+M) \tilde{y}_{t}+N L \tilde{y}=0 \quad \text { in } L^{2}(Q), \\
\tilde{y}(\cdot, 0)=-\tilde{z}(\cdot) \quad \text { in } H_{0}^{1}(G) \cap H^{2}(G) .
\end{gathered}
$$

By (2.5), we have that

$$
\|\tilde{y}(\cdot, t)\|_{0}<\sqrt{k_{m}} \exp \left(-N t c_{l} / k_{m}\right)\|\tilde{z}(\cdot)\|_{1} \cdot
$$

The solution of (3.5), however, is given by $\tilde{y}(\cdot, t)=-E(N t) \tilde{z}(\cdot)$ so that $y(\cdot, t ; \tilde{u})-\tilde{z}(\cdot)=\tilde{y}(\cdot, t)$. Hence, we see that the estimate (3.6) holds for $y\left(\cdot, t ; \tilde{u}_{N}\right)-\tilde{z}(\cdot)$. Therefore, by choosing $N$ such that

$$
\sqrt{k_{m}} \exp \left(-N T c_{l} / k_{m}\right)\|\tilde{z}(\cdot)\|_{1}<\rho
$$

we obtain condition (3.2).

For problem (1.1) we may also establish the following result. 
LEMMA 3.2 If $\tilde{z}$ belongs to $H_{0}^{1}(G) \cap H^{2}(G)$, then the control

$$
u_{\tilde{z}}(\cdot, t)=T^{-1}(1+M) E(t-T) \tilde{z}(\cdot)
$$

is such that $y\left(\cdot, T ; u_{\tilde{z}}\right)=\tilde{z}(\cdot)$.

That Lemma 3.2 is true may be seen by replacing $u$ by $u_{i}$ in (2.1) and performing the integration.

REMARK 3.3. Lemma 3.2 implies that $H_{0}^{1}(G) \cap H^{2}(G) \subset Y(T)$. From the representation equation (2.1), however, it is clear that $Y(T) \subset H_{0}^{1}(G) \cap$ $H^{2}(G)$. Hence, we have the equality of the two subspaces.

THEOREM 3.4. The pseudo-parabolic initial-value problem (1.1) is controllable in the sense that $Y(T)$ is a dense subspace of $L^{2}(G)$.

We now consider the compatibility of the set $N(0, b)$ and $V(Z, \rho)$. First, we specify that

$$
0<\rho<\|Z\|_{0}
$$

This is a reasonable condition in that if $\rho=0$ then the endpoint condition may never be satisfied because $Z$ is only required to belong to $L^{2}(G)$. On the other hand, if $\rho=\|Z\|_{0}$, then $0 \in V(Z, \rho)$, and we will see that this implies that 0 is the solution of (1.6). Furthermore, we note that Theorem 3.4 implies that $V(Z, \rho)$ is nonempty. Hence, $V(Z, \rho)$ is a nonempty closed convex subset of $L^{2}(Q)$.

Now in order that $N(0, b) \cap V(Z, \rho)$ be nonempty, the parameter $b$ must be sufficiently large. Obviously, if it is true that

$$
b>b(Z, \rho)
$$

where

$$
b(Z, \rho)=\left\|u_{(Z, \rho)}\right\|_{0,0}=\underset{u \in V(Z, \rho)}{\operatorname{minimum}}\|u\|_{0,0}
$$

then $U_{\text {ad }}$ is nonempty. That $u_{(Z, \rho)}$ exists follows from the well-known fact that every nonempty closed convex subset of a Hilbert space has an element of minimum norm. Furthermore, condition (3.8) implies $b(Z, \rho)>0$. Another, and perhaps more desirable, way to set $b$ is to find some $\tilde{z}$ in $H_{0}^{1}(G) \cap$ $H^{2}(G)$ close to $Z$. Then, use Lemma 3.1 or Lemma 3.2 to find a control $\tilde{v}$ steering close to $\tilde{z}$ and set $b \geqslant\|\tilde{v}\|_{0,0}$. For convenience, however, we will use (3.9) in our discussion.

We finish this section by demonstrating the existence and uniqueness of the solution of (1.6). To this end, we first observe some properties of the control functional $K(\cdot)$. We may write $K(u)$ in an alternate form from that in (1.5). If we multiply the differential equation in (1.1) by $y(u)$ and integrate over $Q$, we 
obtain

$$
\begin{aligned}
K(u)= & \frac{1}{2} \int_{G}\left[(1+m(x)) y^{2}(x, T ; u)\right. \\
& \left.+\sum_{i, j=1}^{n} \frac{\partial y(x, T ; u)}{\partial x_{i}} m_{i j}(x) \frac{\partial y(x, T ; u)}{\partial x_{j}}\right] d x \\
& +\int_{Q}\left[l(x) y^{2}(x, t ; u)+\sum_{i, j=1}^{n} \frac{\partial y(x, t ; u)}{\partial x_{i}} l_{i j}(x) \frac{\partial y(x, t ; u)}{\partial x_{j}}\right] d x d t
\end{aligned}
$$

(i) $K(u) \geqslant 0$ for all $u$ in $L^{2}(Q)$.

Hence, if $\rho=\|Z\|_{0}$, then $0 \in U_{\mathrm{ad}}$ and $u_{0}=0$.

(ii) $K(\cdot)$ is continuous on $L^{2}(Q)$.

This fact follows from Corollary 2.3 and the inequalities

$$
\begin{aligned}
|K(v)-K(u)| & <\left|(v, y(v)-y(u))_{0,0}\right|+\left|(v-u, y(u))_{0,0}\right| \\
& <C\|v\|_{0,0}\|v-u\|_{0,0}+\|v-u\|_{0,0} C\|u\|_{0,0} \\
& <C\left(\|v\|_{0,0}+\|u\|_{0,0}\right)\|v-u\|_{0,0} .
\end{aligned}
$$

So that $K(v) \rightarrow K(u)$ as $v \rightarrow u$ in $L^{2}(Q)$.

(iii) $K(\cdot)$ is a strictly convex functional on $L^{2}(Q)$.

Given unequal elements $u_{1}$ and $u_{2}$ in $L^{2}(Q)$ and $\theta$ in $(0,1)$, we have

$$
K\left(\theta u_{1}+(1-\theta) u_{2}\right)<\theta K\left(u_{1}\right)+(1-\theta) K\left(u_{2}\right) \text {. }
$$

(iv) $K(\cdot)$ is a weakly lower semicontinuous functional on $L^{2}(Q)$.

Having observed these properties, we have the following theorem.

TheOREM 3.5. Let $\rho$ and $b$ satisfy (3.8) and (3.9). Then problem (1.6) has $a$ unique solution $u_{0}$.

Proof. Since $U_{\mathrm{ad}}$ is defined by (1.4), it is a nonempty closed bounded convex subset of $L^{2}(Q)$. Hence, $U_{\text {ad }}$ is weakly closed and weakly compact. The existence of a solution $u_{0}$ of (1.6) then follows from properties (i) and (iv) of $K(\cdot)$. The uniqueness of $u_{0}$ results from the convexity of $U_{\text {ad }}$ and the strict convexity of $K(\cdot)$.

REMARK 3.6. By considering the first variation of $K(\cdot)$, we may characterize $u_{0}$ by means of the variational inequality

$$
\left(v-u_{0}, y\left(u_{0}\right)\right)_{0,0}+\left(u_{0}, y\left(v-u_{0}\right)\right)_{0,0}>0
$$

for all $v$ in $U_{\text {ad }}$.

4. Regularity of the optimal control. The characterization of $u_{0}$ given by (3.10) is in the form of a variational inequality because of the presence of the control constraints. In this section we prove the existence of positive numbers $\lambda_{1}$ and $\lambda_{2}$, Lagrange multipliers [7], [8] associated with the control constraints 
that enable us to reformulate the original minimization problem over all of $L^{2}(Q)$. A variational identity results in this case and enables us to write a characterization of the optimal control in terms of a system of differential equations. From this characterization we are able to deduce the regularity properties of the optimal control by using the regularity theory for the Dirichlet problem [1], [4], [10], [11].

Here we assume that $Z \in H^{k}(G), k \geqslant 0$ and that $b>b(Z, \rho)$.

LEMMA 4.1. If $b>b(Z, \rho)$, then there exists a control $\bar{u}$ in $L^{2}(Q)$ such that $\|\bar{u}\|_{0,0}<b$ and $\|y(\cdot, T ; \bar{u})-Z(\cdot)\|_{0}<\rho$.

Proof. From the controllability of the Cauchy problem (1.1), we know there exists an element $\bar{v}$ in $L^{2}(Q)$ such that $\|y(\cdot, T ; \bar{v})-Z(\cdot)\|_{0}<\rho$. We now consider convex combinations of $\bar{v}$ with the element $u_{(Z, \rho)}$ defined by (3.10), $u_{\alpha}=\alpha \bar{v}+(1-\alpha) u_{(Z, \rho)}$. Thus,

$$
\begin{aligned}
\left\|y\left(\cdot, T ; u_{\alpha}\right)-Z(\cdot)\right\|_{0}< & \alpha\|y(\cdot, T ; \bar{v})-Z(\cdot)\|_{0} \\
& +(1-\alpha)\left\|y\left(\cdot, T ; u_{(Z, \rho)}\right)-Z(\cdot)\right\|_{0} \\
& <\alpha \rho+(1-\alpha) \rho=\rho .
\end{aligned}
$$

Furthermore, we see that

$$
\begin{aligned}
\left\|u_{\alpha}\right\|_{0,0} & \leqslant \alpha\|\bar{v}\|_{0,0}+(1-\alpha)\left\|u_{(Z, \rho)}\right\|_{0,0} \\
& <\alpha\|\bar{v}\|_{0,0}+(1-\alpha) b
\end{aligned}
$$

Accordingly, there exists $\bar{\alpha}>0$ such that if $\alpha \in(0, \bar{\alpha}]$, then $\left\|u_{\alpha}\right\|_{0,0}<b$. Setting $\bar{u}=u_{\bar{\alpha}}$, we are done.

We now define the functionals on $L^{2}(Q)$

$$
G_{1}(u) \equiv\|y(\cdot, T ; u)-Z(\cdot)\|_{0}^{2}-\rho^{2}
$$

and

and the subsets of $R^{3}$

$$
G_{2}(u) \equiv\|u\|_{0,0}^{2}-b^{2}
$$

$$
K_{1}=\left\{(x, y, z): x \geqslant K(u), y \geqslant G_{1}(u), \text { and } z \geqslant G_{2}(u) \text { for some } u \in L^{2}(Q)\right\}
$$

and

$$
K_{2}=\{(x, y, z): x \leqslant d, y<0, \text { and } z<0\}
$$

where $d=K\left(u_{0}\right)$. The Lagrange multipliers are associated with the direction numbers of a plane that separates the sets $K_{1}$ and $K_{2}$.

We note the convexity and separation properties of the sets. First, we note from Lemma 4.1 that there is a $\bar{u} \in L^{2}(Q)$ satisfying the conditions $G_{1}(\bar{u})<$ 0 and $G_{2}(\bar{u})<0$, simultaneously.

(i) $K_{1}$ and $K_{2}$ are convex subsets of $R^{3}$. 
(ii) int $K_{2} \neq \varnothing$.

(iii) $K_{1} \cap$ int $K_{2}=\varnothing$.

(iv) $\{(d, 0,0)\}=K_{1} \cap K_{2}$.

We prove (iii) since (iv) follows from (iii) and consideration of the various possibilities. Let $(x, y, z) \in K_{1} \cap$ int $K_{2}$. Now the fact that $(x, y, z)$ belongs to $K_{1}$ implies the existence of $\bar{v}$ in $L^{2}(Q)$ such that $x \geqslant K(\bar{v}), y \geqslant G_{1}(\bar{v})$, and $z \geqslant G_{2}(\bar{v})$. On the other hand, the fact that $(x, y, z)$ belongs to int $K_{2}$ implies $K(\bar{v}) \leqslant x<d, G_{1}(\bar{v}) \leqslant y<0$, and $G_{2}(\bar{v}) \leqslant z<0$. But, if $G_{1}(\bar{v})<0$ and $G_{2}(\bar{v}) \leqslant 0$, then $K(\bar{v}) \geqslant d$. Thus $d \leqslant x<d$ which is false. Hence $G_{1}(\bar{v})<0$ and $G_{2}(\bar{v})<0$ both cannot happen. The assumption $(x, y, z) \in$ int $K_{2}$ then implies either $0>y \geqslant G_{1}(\bar{v}) \geqslant 0$ or $0>z \geqslant G_{2}(\bar{v}) \geqslant 0$ both of which are false. We conclude, therefore, that $K_{1} \cap$ int $K_{2}=\varnothing$.

The above properties imply that $K_{1}$ and $K_{2}$ may be separated by a plane. Since the point $(d, 0,0)$ belongs to both sets, the separating plane has the form $\gamma_{0}(x-d)+\gamma_{1} y+\gamma_{2} z=0$. That $\gamma_{0}, \gamma_{1}$, and $\gamma_{2}$ are nonzero can be easily seen by using the fact that $G_{1}(\bar{u})<0$ and $G_{2}(\bar{u})<0$ and considering the various cases. Let $\lambda_{1}=\gamma_{1} / \gamma_{0}$ and $\lambda_{2}=\gamma_{2} / \gamma_{0}$ so that $x+\lambda_{1} y+\lambda_{2} z=d$ is the equation of the separating plane.

Consequently, we may write

$$
\inf _{(x, y, z) \in K_{1}}\left(x+\lambda_{1} y+\lambda_{2} z\right) \geqslant d \geqslant \sup _{(x, y, z) \in K_{2}}\left(x+\lambda_{1} y+\lambda_{2} z\right) .
$$

We show that $\lambda_{1}>0$ and $\lambda_{2} \geqslant 0$. If either $\lambda_{1}$ or $\lambda_{2}$ is negative, say $\lambda_{1}<0$, then the inequality $x+\lambda_{1}(y+n)+\lambda_{2} z \geqslant d>0$, which holds for all $n>0$ if $(x, y, z) \in K_{1}$, clearly produces a contradiction. Hence, $\lambda_{1}$ and $\lambda_{2}$ are nonnegative. To see that $\lambda_{1}>0$, consider only $\|u\|_{0,0} \leqslant b$, that is, only those points $(x, y, 0)$ in $K_{1}$. In this case for $u=0$, we see that $\left(0,\|Z\|_{0}^{2}-\rho^{2}, 0\right)$ belongs to $K_{1}$. Hence, from (4.5), we have $\lambda_{1} \geqslant d /\left(\|Z\|_{0}^{2}-\rho^{2}\right)>0$ so that $\lambda_{1}>0$.

From 4.5, we have in particular

$$
\inf _{u \in L^{2}(Q)}\left[K(u)+\lambda_{1} G_{1}(u)+\lambda_{2} G_{2}(u)\right] \geqslant d .
$$

For the optimal control $u_{0}$, however, $G_{1}\left(u_{0}\right) \leqslant 0$ and $G_{2}\left(u_{0}\right) \leqslant 0$. Thus we see that

$$
d \leqslant K\left(u_{0}\right)+\lambda_{1} G_{1}\left(u_{0}\right)+\lambda_{2} G_{2}\left(u_{0}\right) \leqslant K\left(u_{0}\right)=d .
$$

Defining the Lagrangian $\Lambda(u)=K(u)+\lambda_{1} G_{1}(u)+\lambda_{2} G_{2}(u)$, we have

$$
\min _{u \in L^{2}(Q)} \Lambda(u)=\Lambda\left(u_{0}\right)=K\left(u_{0}\right)=d .
$$

Hence, by taking the first variation of $\Lambda$, we obtain the identity

$$
\begin{aligned}
0= & \left(u_{0}, y(v)\right)_{0,0}+\left(v, y\left(u_{0}\right)\right)_{0,0} \\
& +2 \lambda_{1}\left(y\left(\cdot, T ; u_{0}\right)-Z(\cdot), y(\cdot, T ; v)\right)_{0}+2 \lambda_{2}\left(u_{0}, v\right)_{0,0}
\end{aligned}
$$


for all $v$ in $L^{2}(Q)$. We now introduce the following adjoint Cauchy problems

$$
\begin{gathered}
-(1+M) p_{t}\left(u_{0}\right)+L p\left(u_{0}\right)=u_{0} \text { in } L^{2}(Q), \\
p\left(\cdot, T ; u_{0}\right)=0 \text { in } L^{2}(G),
\end{gathered}
$$

and

$$
\begin{gathered}
-(1+M) q_{t}\left(u_{0}\right)+L q\left(u_{0}\right)=0 \text { in } L^{2}(Q), \\
q\left(\cdot, T ; u_{0}\right)=(1+M)^{-1}\left(y\left(\cdot, T ; u_{0}\right)-Z(\cdot)\right) \quad \text { in } H_{0}^{1}(G) \cap H^{2}(G) .
\end{gathered}
$$

Using (4.10) and (4.11), we may now write (4.9) in the form

$$
\left(y\left(u_{0}\right)+p\left(u_{0}\right)+2 \lambda_{1} q\left(u_{0}\right)+2 \lambda_{2} u_{0}, v\right)_{0,0}=0
$$

for all $v$ in $L^{2}(Q)$. Hence we conclude that

$$
y\left(u_{0}\right)+p\left(u_{0}\right)+2 \lambda_{1} q\left(u_{0}\right)+2 \lambda_{2} u_{0}=0
$$

in $L^{2}(Q)$. We collect these results in the following theorem.

THEOREM 4.2. If $\lambda_{1}$ and $\lambda_{2}$ are Lagrange multipliers associated with the constraints $\|y(\cdot, T ; u)-Z(\cdot)\|_{0} \leqslant \rho$ and $\|u\|_{0,0} \leqslant b$, then the optimal control $u_{0}$ is characterized by the equations (1.1) and (4.10)-(4.12).

It is from (4.12) that we may deduce the regularity of the optimal control, if we know that $\lambda_{2}>0$. Hence, we assume that $\lambda_{2}=0$ and seek a contradiction. Now, using the fact that $y\left(u_{0}\right), p\left(u_{0}\right)$ and $q\left(u_{0}\right)$ are strongly differentiable with respect to $t$, we may use equation (2.2) and analogous results for $p\left(u_{0}\right)$ and $q\left(u_{0}\right)$ to obtain

$$
-(1+M)^{-1} L y\left(u_{0}\right)+(1+M)^{-1} L p\left(u_{0}\right)+2 \lambda_{1}(1+M)^{-1} L q\left(u_{0}\right)=0
$$

in $L^{2}(Q)$. Subtracting (4.13) from the equation obtained by applying $(1+$ $M)^{-1} L$ to (4.12), we see that

$$
(1+M)^{-1} L y\left(u_{0}\right)=0
$$

in $L^{2}(Q)$. Since $(1+M)^{-1} L$ is an isomorphism from $H_{0}^{1}(G) \cap H^{2}(G)$ onto itself, we have $y\left(u_{0}\right)=0$ in $L^{2}(Q)$. But $t \rightarrow y\left(\cdot, t ; u_{0}\right)$ is a continuous mapping of $[0, T]$ into $L^{2}(G)$. Hence, we see that $y\left(\cdot, t ; u_{0}\right)=0$ in $L^{2}(G)$ for each $t$ in $[0, T]$. In particular, we have $y\left(\cdot, T ; u_{0}\right)=0$ in $L^{2}(G)$. However, the constraint, $\|y(\cdot, T ; u)-Z(\cdot)\|_{0} \leqslant \rho$, now implies $\|Z(\cdot)\|_{0} \leqslant \rho$ contrary to the assumption $\|Z(\cdot)\|_{0}>\rho$. Therefore, we conclude that $\lambda_{2}$ is strictly positive. Having proved this, we may easily observe the next result.

THEOREM 4.3. If $u_{0}$ is the optimal control, then $\left\|y\left(\cdot, T ; u_{0}\right)-Z(\cdot)\right\|_{0}=\rho$ and $\left\|u_{0}\right\|_{0,0}=b$. 
Proof. From (4.7) we see that

$$
\lambda_{1} G_{1}\left(u_{0}\right)+\lambda_{2} G_{2}\left(u_{0}\right)=0 .
$$

However, with $G_{1}\left(u_{0}\right)$ and $G_{2}\left(u_{0}\right)$ nonpositive and $\lambda_{1}$ and $\lambda_{2}$ positive, we see that (4.15) implies $G_{1}\left(u_{0}\right)=0$ and $G_{2}\left(u_{0}\right)=0$ yielding the result.

TheOREM 4.4. Let $b>b(Z, \rho)$ and the coefficients in $M$ and $L$ and the boundary of $G$ satisfy the smoothness assumptions in $\S 1$ for $p>2$. Let $Z$ be in $H^{k}(G)$. If $p-2 \geqslant k \geqslant 0$, then

$$
u_{0} \in H^{1}\left(0, T ; H_{0}^{1}(G) \cap H^{k+2}(G)\right) .
$$

If $k \geqslant p-1$, then

$$
u_{0} \in H^{1}\left(0, T ; H_{0}^{1}(G) \cap H^{p}(G)\right)
$$

For $p=\infty$, we record the following corollary.

COROLlARY 4.5. If $b>b(Z, \rho)$ and the boundary of $G$ and the coefficients in $L$ and $M$ are smooth and if $Z$ is in $H^{k}(G)$, then $u_{0}$ is in $H^{1}\left(0, T ; H_{0}^{1}(G) \cap\right.$ $\left.H^{k+2}(G)\right)$.

REMARK 4.6. Because of its dependence upon the property int $U_{\text {ad }} \neq \varnothing$, the above analysis does not apply to the case in which $b=b(Z, \rho)$. We have indicated in (3.9) that there is an element $u_{(Z, \rho)}$ in $L^{2}(Q)$ such that $b(Z, \rho)=$ $\left\|u_{(Z, \rho)}\right\|_{0,0}$. Furthermore, that $u_{(Z, \rho)}$ is unique follows from the convexity of $V(Z, \rho)$. Hence, in this case $U_{\mathrm{ad}}=\left\{u_{(Z, \rho)}\right\}$. Accordingly, $u_{(Z, \rho)}$ is trivially the solution of problem (1.6) for $b=b(Z, \rho)$. We may deduce the regularity of $u_{0}=u_{(Z, \rho)}$ by applying the Lagrange multiplier technique to the problem

$$
\text { minimize }\|u\|_{0,0} \text { subject to }\|y(\cdot, T ; u)-Z(\cdot)\|_{0}<\rho \text {. }
$$

When this is done, we obtain a characterization of $u_{0}$ by means of the system of equations

$$
\begin{gathered}
(1+M) y_{t}\left(u_{0}\right)+L y\left(u_{0}\right)=u_{0} \text { in } L^{2}(Q), \\
y\left(\cdot, 0 ; u_{0}\right)=0 \text { in } L^{2}(G), \\
(1+M) q_{t}\left(u_{0}\right)+L q\left(u_{0}\right)=0 \text { in } L^{2}(Q), \\
q\left(\cdot, T ; u_{0}\right)=(1+M)^{-1}\left(y\left(\cdot, T ; u_{0}\right)-Z(\cdot)\right) \\
\text { in } H_{0}^{1}(G) \cap H^{2}(G), \\
u_{0}+\lambda q\left(u_{0}\right)=0 \text { in } L^{2}(Q) .
\end{gathered}
$$

This yields the following regularity results.

THEOREM 4.7. If $Z$ is in $H^{k}(G)$ and the boundary of $G$ and the coefficients of $L$ and $M$ are sufficiently smooth, then $u_{0}$ is in $H^{1}\left(0, T ; H_{0}^{1}(G) \cap H^{k+2}(G)\right)$. 


\section{REFERENCES}

1. S. Agmon, Lectures on elliptic boundary value problems, Van Nostrand, New York, 1965.

2. A. G. Butkovskiy, Distributed control systems, American Elsevier, New York, 1969.

3. R. W. Carroll and R. E. Showalter, Singular and degenerate Cauchy problems, Academic Press, New York, 1976.

4. A. Friedman, Partial differential equations of parabolic type, Prentice-Hall, Englewood Cliffs, N. J., 1964.

5. E. Hille and R. S. Phillips, Functional analysis and semi-groups, Amer. Math. Soc., Colloq. Publ., vol. 31, Amer. Math Soc., Providence, R. I., 1957.

6. J. L. Lions, Optimal control of systems governed by partial differential equations (translated by S. K. Mitter), Springer-Verlag, New York, 1971.

7. D. G. Luenberger, Optimization by vector space methods, Wiley, New York, 1969.

8. R. T. Rockafellar, Convex analysis, Princeton Univ. Press, Princeton, N. J., 1972.

9. D. L. Russell, Controllability and stability theory for linear partial differential equations: Recent progress and open questions, MRC Technical Summary Report \#1700, Mathematics Research Center, Madison, Wisconsin, 1976.

10. R. E. Showalter and T. W. Ting, Pseudo-parabolic partial differential equations, SIAM J. Math. Anal. 1 (1970), 1-26.

11. F. Treves, Basic linear partial differential equations, Academic Press, New York, 1974.

12. L. W. White, Control problems governed by pseudo-parabolic partial differential equations, Ph.D. Thesis, University of Illinois at Urbana-Champaign, 1977.

13. K. Yosida, Functional analysis, Springer-Verlag, Berlin, 1974.

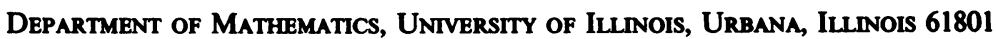

Current address: Department of Mathematics, University of Oklahoma, Norman, Oklahoma 73019 